\title{
High spectral purity optical source stabilized on a fiber ring resonator
}

\author{
Gilles Bailly, Olivier Llopis Member, IEEE, Arnaud Fernandez
}

\begin{abstract}
This paper demonstrates the stabilization of an external cavity semiconductor laser at $1550 \mathrm{~nm}$ on an ultra-high $Q$ factor fiber ring resonator (FRR). Based on a Pound-Drever-Hall technique, our system is optimized in order to take into account the properties of the FRR which features a large $Q$ factor $\left(2 \times 10^{9}\right)$ but a relatively small free spectral range $(2 \mathrm{MHz})$. The measured performances of the stabilized laser shows a frequency noise level as low as $0.4 \mathrm{~Hz}^{2} / \mathrm{Hz}$ in the $1 \mathrm{kHz}$ to $10 \mathrm{kHz}$ range and a linewidth lower than $30 \mathrm{~Hz}$ for $5 \mathrm{~ms}$ integration time.
\end{abstract}

Index Terms - Semiconductor laser, Optical resonator, Phase noise, Linewidth, Allan deviation

\section{INTRODUCTION}

$\mathrm{H}^{\prime}$ IGH spectral purity optical sources are required for different application domains such as optical sensors, microwave optics, time and frequency metrology or coherent communications. Optical fiber sensors rely on stable frequency sources in the $1 \mathrm{~Hz}$ to $1 \mathrm{kHz}$ range [1,2]. For LiDAR applications, the requirements depend on the target type and the measurement performed (distance or velocity). However, the optical source phase noise is always a limiting factor [3]. High spectral purity lasers are also used in optical clocks, in order to get simultaneously long and short-term frequency stability. Combined with an optical frequency comb, they will perform high quality optical frequency synthesis. They are also useful to check the performance of laser phase noise measurement systems.

In terms of frequency stability, the best lasers are stabilized on ultra-low expansion (ULE) glass or silicon Fabry-Perot resonators [4-7]. However, these sources are generally expensive and only deliver a fixed frequency signal. Such resonators can be made compact [7], but the application of this approach to embedded systems has still to be demonstrated. A good compromise between frequency stability and system size can be obtained by using small size resonators such as whispering gallery mode (WGM) resonators [8] or integrated spiral resonators [9]. The high spectral purity laser given in [8] is highly compact, thanks to the use of direct optical feedback to stabilize the laser on the resonator. However, the coupling of WGM resonators to an optical waveguide is still difficult and it is hard to provide such a technology at low cost. Finally, if one seek for high spectral purity and tunability, a solution lies in the

Manuscript received Dec. 2019.

G. Bailly (gilles.bailly@laas.fr), O. Llopis (1lopis@laas.fr) and A. Fernandez (arnaud.fernandez@laas.fr) are with LAAS-CNRS, Université de Toulouse, CNRS, Toulouse, France. A. Fernandez is also with UPS (Université Paul Sabatier), Toulouse, France.

This work has been partially supported by the Centre National d'Etudes Spatiales (CNES). stabilization of the laser on a frequency discriminator [10-12]. The discriminator mixes on a photodiode a reference signal with a delayed signal from a fiber spool. The laser source is a fiber laser [10,11] or a solid state laser [12], although it should be possible to use this approach with a semiconductor laser. These systems are quite bulky, due to the use of large fiber delay.

In order to get a compact, easy to assemble and low phase noise optical source, a good solution is to stabilize a semiconductor laser on a fiber resonator. Such an approach has already been proposed with a fibered Fabry-Perot resonator and a DFB laser [13]. In this paper, we propose a similar approach on a higher $\mathrm{Q}$ factor resonator realized with a fiber ring resonator (FRR). The optimization of the Pound-Drever-Hall stabilization loop, together with the intrinsic laser and resonator performance, lead to a ten hertz range linewidth source which surpasses classical commercially available lasers such as external cavity semiconductor lasers or fiber lasers.

\section{OPTICAL SOURCE DESIGN}

The key optical elements of our source are the semiconductor laser and the FRR. The laser is an external cavity laser in butterfly package (RIO Planex @ $1.55 \mu \mathrm{m}$ ) which can be coupled to an ultra high $\mathrm{Q}$ cavity because of its already reduced linewidth $(\sim 10 \mathrm{kHz})$. The only drawback of the RIO laser resides in its reduced tunability compared to a classical DFB laser, which is in the range of $30 \mathrm{GHz}$ for a $20^{\circ} \mathrm{C}$ temperature change (including some mode hopping). The FRR depicted in Fig. 1 is composed of two identical low loss optical couplers showing $8 \%$ coupling factor, $100 \mathrm{~m}$ PM fiber and an intracavity optical isolator to prevent the generation of Brillouin backscattering [14]. It features a measured $\mathrm{Q}$ factor of about $2 \times 10^{9}$, a free spectral range (FSR) of $2 \mathrm{MHz}$ and was originally designed for optoelectronic oscillator stabilization.

As the laser linewidth is narrow enough compared to the FRR's resonance width (100 kHz in our case), laser injection at input port results immediately in a FRR resonance shift due to self-heating. Indeed the $7 \mathrm{~dB}$ intra-cavity power enhancement factor results in more than $13 \mathrm{dBm}$ intra-cavity power when the resonator is fed with $6 \mathrm{dBm}$ optical power [14].

Therefore, a feedback must be set-up to stabilize the laser frequency on a FRR resonance frequency. The laser frequency noise will be improved in the locking bandwidth by the ultrahigh Q factor of the resonator (compared to the intrinsic Q factor of the free running laser). For an easy control of the feedback loop gain and bandwidth parameters, a Pound-DreverHall (PDH) scheme has been chosen [15]. As depicted in Fig. 2, 
the laser stabilization is performed on the through port of the resonator in order to use an out of band modulation (modulation frequency greater than the FRR resonance half-bandwidth). The resonant signal leaving the cavity is collected on the drop port. A $\mathrm{LiNbO}_{3}$ phase modulator allows the laser modulation at 19.5 MHz, which is detected and contributes to the error signal generation after the mixer (see Fig. 2). This modulation frequency is high enough compared to the correction bandwidth. The laser frequency correction is realized using the dedicated modulation input of the ultra-low noise current controller (SMC11 from Sisyph), which provides a transfer of $1 \mathrm{~mA} / \mathrm{V}$ from $\mathrm{DC}$ to $20 \mathrm{MHz}$ or $100 \mathrm{MHz} / \mathrm{V}$ considering the RIO laser sensitivity. At the end, the system could be made compact through the integration of the electronic circuit.

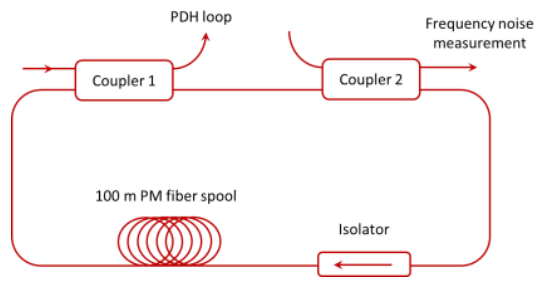

Fig. 1. Schematic of the fiber ring resonator built with two low loss $8 \%$ PM couplers, a low loss PM isolator and a $100 \mathrm{~m}$ PM fiber spool. The measured Q factor is $2 \times 10^{9}$ and the resonator is relatively flat and occupies a $10 \mathrm{~cm}^{2}$ area.

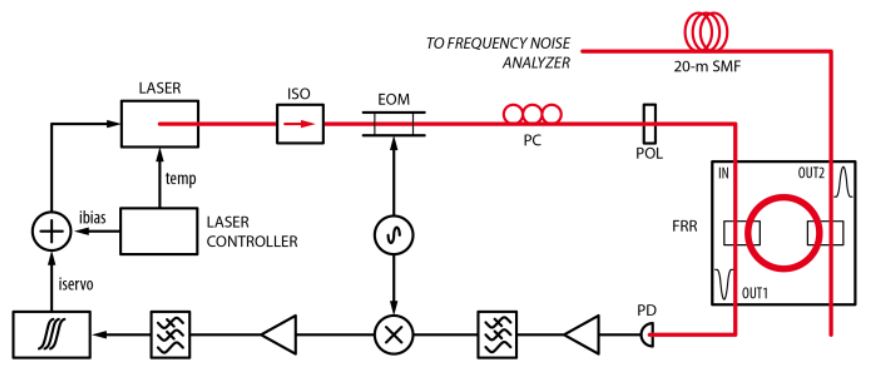

Fig. 2. Global schematic of the RIO laser stabilization set-up on a fiber ring resonator using the PDH technique. A polarization controller and a polarizer allows a proper use of the full PM resonator. The optical signal is modulated in phase with the electro-optic modulator (EOM) and the resonator impact on the generated sidebands is recovered after the photodiode (PD) and the RF mixer.

To get high performance in terms of frequency noise, the PDH loop must be carefully tuned to get a high correction gain over the largest possible bandwidth. Nonetheless, the closed-loop bandwidth is actually limited by the resonator's FSR, which introduces zeroes in the spectrum of the error signal. Since these zeroes occur at multiples of the FSR, the first zero is placed at $2 \mathrm{MHz}$, limiting therefore the available bandwidth between $100 \mathrm{kHz}$ and $1 \mathrm{MHz}$. In order to get the maximum loop gain in this particular situation, a high-order compensator has been developed to provide tight control of the laser despite this reduced bandwidth. Indeed, two additional integrators are added to an analog PID (proportional-integral-derivative) in order to increase the low-frequency loop-gain while the D-term insures stability. Its phase-lead has been tuned to prevent the degradation of the phase margin due to the propagation delay and the FSR's zero. Thereby, an open-loop gain of $60 \mathrm{~dB}$ at $10 \mathrm{kHz}$ and a bandwidth of $600 \mathrm{kHz}$ have been both achieved using the PI3D compensator. This high loop-gain, combined with the high-stability of the laser controller, is sufficient to maintain the laser locked on one resonator mode during hours. Longer operation can also be obtained by appropriate feedback on the laser temperature controller (and a thermal control of the FRR could also be considered to this purpose).

\section{FREQUENCY NOISE CHARACTERIZATION OF THE STABILIZED SOURCE}

The characterization of a high spectral purity laser can be performed through the comparison with a reference laser, if such a laser is available at the same frequency. If the device under test (DUT) is at a different frequency, the reference can be transferred close to this frequency using an optical comb. However, it may be hard to get low phase noise performance far from the carrier in this experiment. A simpler approach is the delay line frequency discriminator. Of course, the stability and sensitivity of the discriminator must be carefully optimized to get a measurement noise floor better than the DUT noise.

To this purpose, we use a Michelson type self-heterodyne frequency discriminator, with a $2 \mathrm{~km}$ SMF fiber spool on the delayed arm. The system is described in references $[16,17]$ and the noise floor is evaluated [17] with two identical fiber spools in both arms. Mechanically induced spurs in the $10 \mathrm{~Hz}-1 \mathrm{kHz}$ range was the main drawback with this former setup. It has been improved as it is now mounted in a rack with a protection from acoustic and mechanical vibrations. The $80 \mathrm{MHz}$ oscillator, which is used to get the intermediate frequency through the acousto-optic modulator in the reference arm, has been replaced by a low phase noise synthesizer, since it was a limiting factor at low offset frequencies (1 to $10 \mathrm{~Hz}$ ) [17].

At the output of this system, the laser optical frequency noise is transposed into RF phase noise around $80 \mathrm{MHz}$, which is measured using a signal source analyzer (Keysight, E5052B). The optical frequency noise spectrum is computed from the measured RF phase noise by using the following equation,

$$
S_{v}(f)_{\text {in } H z^{2} / H z}=\frac{f^{2}}{2 \sin ^{2}(\pi f \tau)} 10^{\frac{L_{R F}(f)}{10}}
$$

with $f$ being the offset frequency, $L_{R F}(f)$ the radio frequency single sideband (SSB) phase noise in $\mathrm{dBc} / \mathrm{Hz}$ and $\tau$ the delay between the two arms of the discriminator. $\tau$ is calibrated through the measurement of the first null on the spectrum ( $\tau=10.05 \mu \mathrm{s}$ in this system). The laser SSB phase noise $L(f)$ is computed from $S_{v}(f)$ using the following equation,

$$
L(f)=10 \log \left(\frac{S_{v}(f)}{2 f^{2}}\right)=10 \log \left(\frac{S_{\varphi}}{2}\right)
$$

The measurement results are depicted in Fig. 3 and 4. In Fig. 3, the SSB phase noise $L(f)$ of the stabilized laser is plotted (blue line) and compared to the measurement system noise floor (green dotted line). The noise floor has been measured by adding another $2 \mathrm{~km}$ fiber spool in the other arm of the discriminator [17], to cancel the laser phase noise detection while keeping the other noise sources. It is clear that the measurement system noise floor is reached at low frequency offsets $(f<30 \mathrm{~Hz})$ and also in the $200 \mathrm{~Hz}$ to $1 \mathrm{kHz}$ range. Below $1 \mathrm{kHz}$, it is thus difficult to determine whether the measured 
performance is attributed to the laser or the measurement system. Above $20 \mathrm{kHz}$, the phase noise increases again, because the correction bandwidth limit is reached and then it drops again, probably due to the filtering effect induced by the optical resonator bandwidth.

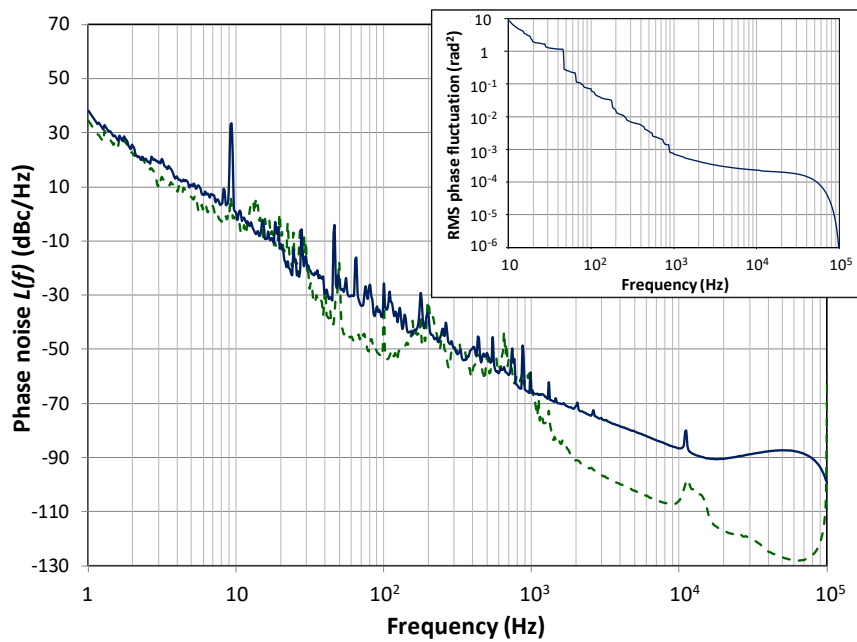

Fig. 3. SSB phase noise spectral density $L(f)$ of the stabilized semiconductor laser (blue continuous line). Comparison with the measurement system noise floor (green doted line). The inset shows the RMS phase noise $\Delta \varphi^{2}$ integrated down from $100 \mathrm{kHz}$ maximum frequency.

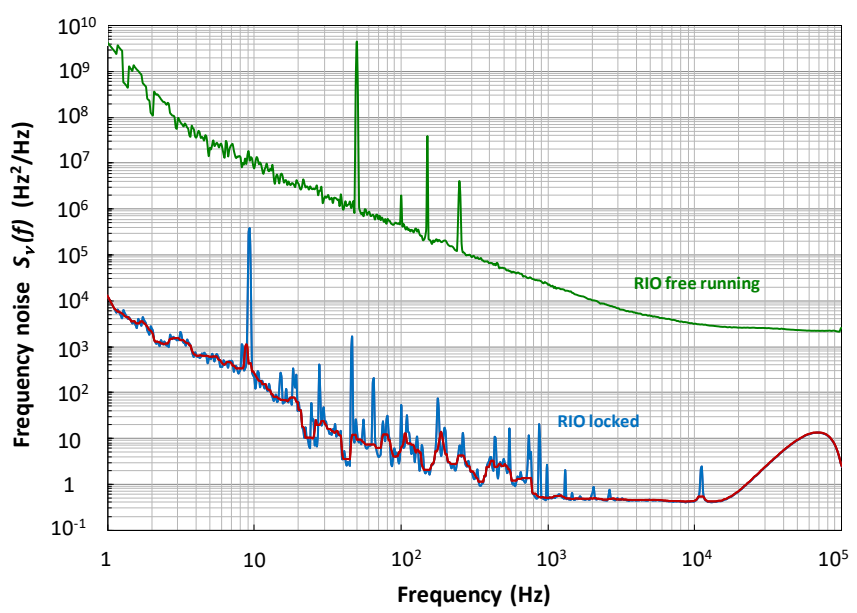

Fig. 4. Frequency noise spectral density $S_{v}$ of the stabilized laser: raw data (blue) and with spurious peaks removed (red). Comparison with the free running laser (green curve).

In Fig. 4, the frequency noise spectral density $S_{v}(f)$ of the same laser (blue curve) is compared to the one of the free running RIO laser (green curve). Between $1 \mathrm{kHz}$ and $20 \mathrm{kHz}$, the locked laser frequency noise drops down to about $0.4 \mathrm{~Hz}^{2} / \mathrm{Hz}$. Closer to the carrier $(f<1 \mathrm{kHz})$, the large number of spurs is typical of vibration induced noise. These vibrations are probably located on the fiber resonator, as they are not all observed on the measurement system noise floor data (Fig. 3). They are removed through fitting (red curve) for further investigations (see paragraph IV).

The short-term frequency noise performance of the stabilized laser compares well with other high quality optical sources. As an example, a frequency noise in the range of $1 \mathrm{~Hz}^{2} / \mathrm{Hz}$ at $1 \mathrm{kHz}$ offset has been measured on a WGM resonator stabilized laser [8] or on a compact ULE resonator stabilized laser [7].

\section{ALLAN DEVIATION, INTEGRATED PHASE NOISE AND LINEWIDTH}

Very often in optics, the performance of a source is specified in terms of linewidth. However, this parameter is not always correctly defined for high spectral purity sources. For many applications (ex: telecom.), lasers are noisy and only the very short-term frequency stability is of interest. The linewidth is then approximated from the white frequency noise data far away from the carrier with the Lorentzian spectrum model. In this special case, the linewidth is independent of the integration time and computed from the frequency noise spectral density multiplied by $\pi$. However, this approximation is totally invalid for high spectral purity lasers or any other source showing a linewidth in the range of the $1 / \mathrm{f}$ noise (i.e. $10 \mathrm{kHz}$ or lower).

The phase noise spectrum $L(f)$ (Fig. 3) gives a different information from the frequency noise $S_{v}(f)$. For high spectral purity sources and at offset frequencies where the phase noise is low enough compared to the carrier (small modulation index), this spectrum represents the ratio between the noise sidebands and the carrier. It is thus a plot of the spectrum "wings" (and this is why the phase noise plot is the main parameter used to assess for sources quality in the RF domain). However, closer from the carrier, when the noise comes close to $0 \mathrm{dBc} / \mathrm{Hz}$, the small signal approximation for noise modulation does not hold. To get an information on the carrier spectral width, it is possible to integrate the phase noise spectral density $S_{\varphi}(f)$ from the offset frequency $f_{c}$ up to the measurements maximum offset frequency [5].

$$
\int_{f_{c}}^{\infty} S_{\varphi}(f) d f=1 \mathrm{rad}^{2}
$$

When this integral equals approximately $1 \mathrm{rad}^{2}$, the total power due to phase fluctuation in each sideband equals the one of the carrier in the central region $\left[v_{0}-v_{c}, v_{0}+v_{c}\right]$. This can be understood using the sinusoidal phase modulation approximation and the corresponding Bessel's function decomposition of the spectrum. In this case, the two first lateral sidebands are at the level of the carrier when the phase modulation maximal excursion is $\Delta \varphi_{\max }=1.435$, or equivalently when $\Delta \varphi_{R M S}=1.435 / \sqrt{2}=1.015 \approx 1$ (a general demonstration would be out of the scope of this paper).

As shown in the inset of Fig. 3 for our laser, the integrated phase noise crosses the $1 \mathrm{rad}^{2}$ level at $30 \mathrm{~Hz}$. This corresponds to a total short-term linewidth of $60 \mathrm{~Hz}$. This value must be considered as an upper value because the spurious peaks are contributing to the integral and the measured data in this frequency range are close to the measurement noise floor.

Allan deviation is another parameter which is popular in time $\&$ frequency field. It is mainly used for long-term $(\tau>1 \mathrm{~s})$ frequency stability characterization, but it can also be computed for short-term fluctuations from the phase noise or frequency noise data. The interest of such a plot is that when the Allan deviation is computed in natural value (in $\mathrm{Hz}$ ) instead of relative value, it represents the laser average frequency deviation versus the integration time. It is thus a plot of the laser linewidth [13] versus the integration time. 
The Allan standard deviation $\sigma_{v}$ can be computed using two different techniques from the laser frequency noise. The first technique involves a fit of the frequency noise curve into various linear segments, each corresponding to a noise contribution: random walk frequency noise $\left(S_{v} \sim 1 / \mathrm{f}^{2}\right)$, flicker frequency noise $\left(S_{v} \sim 1 / \mathrm{f}\right)$, white frequency noise, flicker phase noise $\left(S_{v} \sim \mathrm{f}\right)$, white phase noise $\left(S_{v} \sim \mathrm{f}^{2}\right)$. For each of these contributions, a corresponding slope can be computed in the Allan variance plot using analytical formulas [18]. However, this approach is sensitive to the fitting precision of the spectrum, which is very often not good enough because of the limited choice in spectrum slopes. The second technique consists in a numerical integration of the frequency noise spectrum [18], which is performed directly on the measured $S_{v}$ data using equation (4).

$$
\sigma_{v}^{2}\left(\tau_{k}\right)=\sum_{n=1}^{N} 2 S_{v}\left(f_{n}\right)\left(\frac{\sin ^{2}\left(\pi \tau_{k} f_{n}\right)}{\pi \tau_{k} f_{n}}\right)^{2} \Delta f_{n}
$$

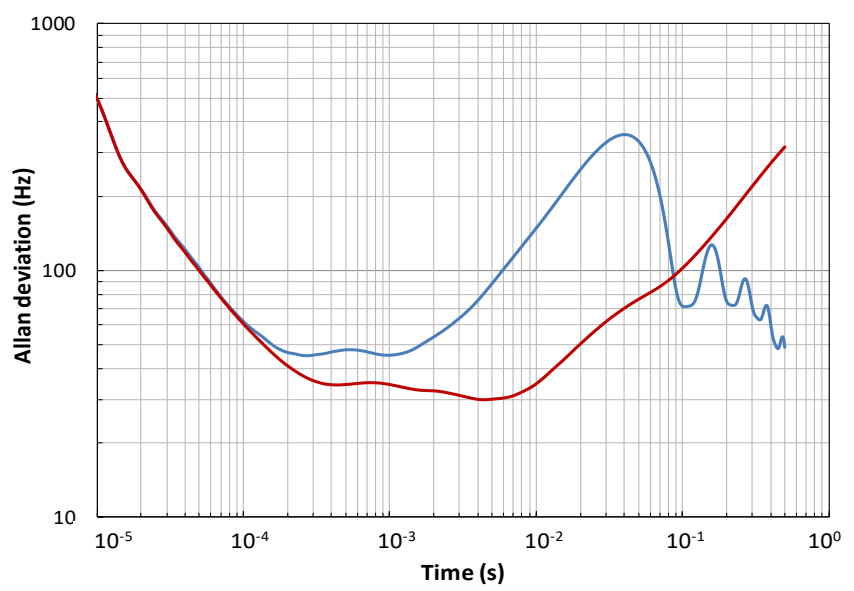

Fig. 5. Allan standard deviation $\sigma_{v}$ (in $\mathrm{Hz}$ ) computed from the measured frequency noise spectral density $S_{\nu}$ using raw data (blue) and fitted $S_{\nu}$ spectrum (red). This plot also gives the laser linewidth versus the integration time.

A direct integration of the $S_{v}$ raw data (Fig. 4 - blue curve) may lead to some unphysical behavior on the Allan deviation (Fig. 5 - blue curve). The spurs on the $S_{v}$ spectrum have a strong influence on the computed $\sigma_{v}$, and it is also the case of spectrum windowing, particularly at low frequency offsets. Therefore, the spurious peaks have been numerically limited and the lower end of the spectrum has been extended down to $0.1 \mathrm{~Hz}$ using a $\frac{1}{f^{\alpha}}$ power law fitting. The Allan deviation computed from these pre-processed data shows a minimum for integration time between $0.3 \mathrm{~ms}$ and $10 \mathrm{~ms}$ and, for longer integration time, the laser frequency drift again, as expected for such a nonthermally controlled source (Fig. 5 - red curve). In terms of performance, this plot shows that the minimum linewidth is $30 \mathrm{~Hz}$, which is obtained at about $5 \mathrm{~ms}$ integration time. Again, because of the proximity of the measurement noise floor, it has to be considered as an upper value for the laser linewidth.

\section{CONCLUSION}

A high spectral purity laser and its characterization technique have been presented. The results are given in terms of phase noise, frequency noise spectra and Allan deviation. The Allan deviation has been used to estimate the laser linewidth versus the integration time. This stabilized source is based on a semiconductor laser and an easy to assemble fiber resonator. It could be made compact through the integration of the electronic part of the PDH stabilization loop. With a frequency noise level of $0.5 \mathrm{~Hz}^{2} / \mathrm{Hz}$ at $1 \mathrm{kHz}$ offset, this optical source will be highly efficient in applications in which the short-term frequency stability is the main parameter of interest.

\section{REFERENCES}

[1] L. Stolpner, S. Lee, S. Li, A. Mehnert, P. Mols, S. Siala, "Low noise Planar External Cavity Laser for Interferometric Fiber Optic Sensors", $19^{\text {th }}$ Int. Conf. on Optical Fibre Sensors, Proc. of SPIE, Vol. 7004, 700457, (2008).

[2] R.E. Bartolo, A. Tveten, C.K. Kirkendal, "The quest for inexpensive, compact, low phase noise laser sources for fiber optic sensing applications", $20^{\text {th }}$ Int. Conf. on Optical Fibre Sensors, Proc. of SPIE, Vol. 7503, 750370 (2009).

[3] E. Dale, W. Liang, D. Eliyahu, A.A. Savchenkov, V.S. Ilchenko, A.B. Matsko, D. Seidel, L. Maleki, "Ultra-Narrow Line Tunable Semiconductor Lasers for Coherent LIDAR Applications" Imaging and Applied Optics 2014, OSA Technical Digest (2014).

[4] T. Kessler, C. Hagemann, C. Grebing, T. Legero, U. Sterr, F. Riehle, M.J. Martin, L. Chen, J. Ye, "A sub-40 mHz linewidth laser based on a silicon single-crystal optical cavity," Nature Photonics, vol. 6, pp. 687692, Sept. 2012.

[5] D. G. Matei, D. G. Matei, T. Legero, S. Häfner, C. Grebing, R. Weyrich, W. Zhang, L. Sonderhouse, J. M. Robinson, J. Ye, F. Riehle, U. Sterr, "1.5 $\mu \mathrm{m}$ Lasers with Sub-10 mHz Linewidth," Physical Review Letters, 118, 263202, June 2017.

[6] J. Alnis, A. Matveev, N. Kolachevsky, Th. Udem, T. W. Hänsch, "Subhertz linewidth diode lasers by stabilization to vibrationally and thermally compensated ultralow-expansion glass Fabry-Pérot cavities," Phys. Rev. A 77, 053809, May 2008.

[7] A. Didier, J. Millo, B. Marechal, C. Rocher, E. Rubiola, R. Lecomte, M. Ouisse, J. Delporte, C. Lacroûte, Y. Kersalé, "Ultracompact reference ultralow expansion glass cavity," Applied Optics, Vol. 57, Issue 22, pp. 6470-6473, Aug. 2018.

[8] W. Liang, V. S. Ilchenko, D. Eliyahu, A. A. Savchenkov, A. B. Matsko, D. Seidel, L. Maleki, "Ultralow noise miniature external cavity semiconductor laser," Nature Com., Vol. 6, Article 7371, June 2015.

[9] H. Lee, M.-G. Suh, T. Chen, J. Li, S. A. Diddams, K. J. Vahala, "Spiral resonators for on-chip laser frequency stabilization," Nature Com., vol. 4, Article 2468, Sept. 2013.

[10] C. Spiegelberg, J. Geng, Y. Hu, Y. Kaneda, S. Jiang, N. Peyghambarian, "Low-noise narrow linewidth fiber laser at $1550 \mathrm{~nm}$ (June 2003)," $J$. Lightw. Technol., vol. 22, no. 1, pp. 57-62, Jan. 2004.

[11] H. Jiang, F. Kéfélian, P. Lemonde, A. Clairon, G. Santarelli, "An agile laser with ultra-low frequency noise and high sweep linearity," Opt. Express 18, 3284-3297, Feb. 2010.

[12] V. Crozatier, G. Gorju, F. Bretenaker, J.L. Le Gouët, I. Lorgeré, C. Gagnol, E. Ducloux, "Phase locking of a frequency agile laser," Appl. Phys. Lett. 89, 261115 (2006).

[13] A. Hallal, S. Bouhier, F. Bondu, "Frequency stabilization of a laser tunable over $1 \mathrm{THz}$ in an all fibered system," IEEE Photonics Tech. Letters, vol. 28, no. 11, pp. 1249-1252, June 2016.

[14] K. Saleh, O. Llopis, G. Cibiel, "Optical scattering induced noise in fiber ring resonators and optoelectronic oscillators," J. Lightw. Technol. 31, 1433-1446 (2013).

[15] R. W. P. Drever, J. L. Hall, F. V. Kowalski, J. Hough, G. M. Ford, A.J. Munley, H. Ward, "Laser phase and frequency stabilization using an optical resonator," Appl. Phys. B (1983).

[16] O. Llopis, P. H. Merrer, H. Brahimi, K. Saleh, P. Lacroix, "Phase noise measurement of a narrow linewidth CW laser using delay line approaches," Opt. Lett. 36, 2713-2715 (2011).

[17] O. Llopis, Z. Abdallah, V. Auroux, A. Fernandez, "High spectral purity laser characterization with a self-heterodyne frequency discriminator," Proc. of the IEEE Int. Freq. Control Symp. \& EFTF, Denver, CO, 2015, pp. 602-605.

[18] J. Rutman, F. L. Walls, "Characterization of frequency stability in precision frequency sources," Proc. of the IEEE, vol. 79, no. 7, pp. 952960, July 1991 